\title{
A novel dopant for spiro-OMeTAD towards efficient and stable perovskite solar cells
}

\author{
Zhipeng Lin ${ }^{1,2,3}$, Jing $\mathrm{Li}^{2}$, Hengyi $\mathrm{Li}^{2}$, Yanping $\mathrm{Mo}^{2}$, Junye $\mathrm{Pan}^{2}$, Chao Wang ${ }^{2}$, Xiao-Li Zhang ${ }^{4}$, \\ Tongle $\mathrm{Bu}^{5^{*}}$, Jie Zhong ${ }^{1,2}$, Yi-Bing Cheng ${ }^{1,2}$ and Fuzhi Huang ${ }^{2 *}$
}

\begin{abstract}
N$-di-4-methoxyphenylamino)9,9'-spirobifluorene (spiro-OMeTAD), as the most commonly used hole transport material (HTM), plays a significant role in the normal structured (n-i-p) high-efficiency perovskite solar cells (PSCs). In general, it is prepared by a halogen solvent (chlorobenzene, CBZ) and needs an ion dopant (lithium bis (trifluoromethanesulfonyl)imide, Li-TFSI) to improve its conductivity and hole mobility. However, such a halogen solvent is not environmentally friendly and the widely used LiTFSI dopant would affect the stability of PSCs. Herein, we develop a non-halogen solvent-tetrahydrofuran (THF)-prepared spiro-OMeTAD solution with a new p-type dopant, potassium bis(fluorosulfonyl)imide (K-FSI), to apply into PSCs. By this strategy, high-hole-mobility spiro-OMeTAD film is achieved. Meanwhile, the potassium ions introduced by diffusion into perovskite surface passivate the interfacial defects. Therefore, a hysteresis-free champion PSC with an efficiency of $21.02 \%$ is obtained, along with significantly improved stability against illumination and ambient conditions. This work provides a new strategy for HTMs toward hysteresis-free high-efficiency and stable PSCs by substituting dopants.
\end{abstract}

Keywords: perovskite solar cells, spiro-OMeTAD, K-FSI, hysteresis-free

\section{INTRODUCTION}

Organic-inorganic metal halide perovskite solar cells (PSCs) have been regarded as one of the most promising photovoltaics due to their high power conversion efficiency (PCE) and low-cost production, attracting more and more attention in recent years [1]. They exhibit in- comparable rapid progress with the PCE increasing from initially $3.8 \%$ to $25.5 \%$ this decade $[2,3]$, owing to the outstanding properties of perovskite absorber such as high optical absorption coefficients [4], long charge diffusion length $[5,6]$, tunable band gaps, and further careful modifications $[7,8]$. For commercialization, PSCs are required to improve their stability. Besides perovskites, hole transport materials (HTMs) conducting the extraction and collection of photo-generated holes, also greatly affect the stability of devices. Different molecular structures of HTMs as well as additives have a great influence on the device performance and stability [9-11].

In a normal structure (n-i-p) of PSC, 2,2',7,7'-tetrakis( $N, N$-di-4-methoxyphenylamino)-9,9'-spirobifluorene (spiro-OMeTAD) is the most widely used HTM. In general, dopants are needed to improve the hole mobility because the pristine spiro-OMeTAD shows poor electrical properties [12]. For example, lithium bis(trifluoromethanesulfonyl)imide (Li-TFSI) is added to accelerate the oxidation of spiro-OMeTAD [13], and 4-tertbutylpyridine $(t-\mathrm{BP})$ is used to not only enable an effective $\mathrm{p}$-doping through interface interaction between $t$-BP and the perovskite layer but also increase the solubility of Li-TFSI in the spiro-OMeTAD solution $[14,15]$. However, these standard bi-dopants of Li-TFSI and $t$-BP have been identified to conjointly destroy the morphology of hole transport layers (HTLs) and long-term stability due to the hygroscopicity of Li-TFSI and the low boiling point of $t$ BP [16-18]. In the case of exposure to the ambient, the evaporation of $t$-BP leads to aggregation and hydration of Li-TFSI, which eventually results in the destruction of the PSC devices $[15,19]$. Hence, several groups utilized hy-

\footnotetext{
${ }^{1}$ Foshan Xianhu Laboratory of the Advanced Energy Science and Technology Guangdong Laboratory, Foshan 528216, China

${ }^{2}$ State Key Laboratory of Advanced Technology for Materials Synthesis and Processing, Wuhan University of Technology, Wuhan 430070, China

${ }^{3}$ International School of Materials Science and Engineering, Wuhan University of Technology, Wuhan 430070, China

${ }^{4}$ School of Materials Science and Engineering, Zhengzhou University, Zhengzhou 450001, China

${ }^{5}$ Energy Materials and Surface Sciences Unit, Okinawa Institute of Science and Technology Graduate University, Okinawa 904-0495, Japan

*Corresponding authors (emails: tonglebu@163.com (Bu T); fuzhi.Huang@whut.edu.cn (Huang F))
} 
drophobic salts as dopants of spiro-OMeTAD to improve the device performance and stability. Seo et al. [20] replaced Li-TFSI with $\mathrm{Zn}$-TFSI ${ }_{2}$, leading to a higher PCE over $22 \%$ and improved illumination stability with nonmigration of zinc ions in HTL. Li et al. [21] developed high-performance PSCs based on copper salts-doped spiro-OMeTAD, suppressing its aggregation and crystal-

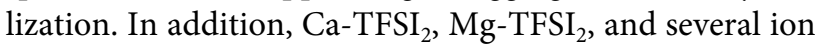
liquids are alternative dopants as well [22-24]. These studies promote the PCE and stability mostly by acquiring superior hole transport and ambient resistance capacity. Actually, as the spiro-OMeTAD is deposited on the top of perovskite, it will alter the interface properties, and thus not only improve the hole extraction but also improve the stability related to the interface, such as hysteresis. Furthermore, the hypertoxicity of chlorobenzene (CBZ), the solvent for spiro-OMeTAD, is another challenge in the PSC fabrication technology. It is desirable to develop environment-friendly solvents in place of CBZ before commercialization.

Herein, we employ a new dopant of potassium bis (fluorosulfonyl)imide (K-FSI) and a non-halogenated solvent of tetrahydrofuran (THF) to prepare spiroOMeTAD HTLs. In general, hysteresis in a normal planar structured device is eliminated by passivating the bottom interface of electron transport layer (ETL) and perovskite film. However, by this approach, suppressed hysteresis is realized through the modification of the top interface. As a result, PSCs based on the K-FSI dopant achieved a champion PCE of $20.94 \%$ for reverse scan (RS) and $21.02 \%$ for forward scan (FS), versus the PCE of Li-TFSIdoped device, $19.75 \%$ for RS and $16.37 \%$ for FS. Characterizations demonstrate that part of $\mathrm{K}^{+}$ions incorporated in the HTL are prone to migrating to the HTL/perovskite interface to passivate surface defects according to the strong interaction between $\mathrm{K}$ and $\mathrm{Br} / \mathrm{I}$. Further, the passivated device shows improved stability under continuous illumination and humid conditions.

\section{EXPERIMENTAL SECTION}

\section{Materials}

Unless specified, all chemicals were purchased from Alfa Aesar or Sigma-Aldrich and used as received. $\mathrm{SnCl}_{2} \cdot 2 \mathrm{H}_{2} \mathrm{O}$ was purchased from Aladdin. Lead iodide $\left(\mathrm{PbI}_{2}\right)$ was purchased from TCI. Methylammonium bromine (MABr), lead bromine $\left(\mathrm{PbBr}_{2}\right)$, formamidinium iodide (FAI) and spiro-OMeTAD were purchased from Xi'an Polymer Light Technology Corp. K-FSI was purchased from Ark Pharm, Inc.

\section{Preparation of perovskite precursor}

The $\mathrm{Cs}_{0.05}\left(\mathrm{FA}_{0.85} \mathrm{MA}_{0.15}\right)_{0.95} \mathrm{~Pb}\left(\mathrm{I}_{0.85} \mathrm{Br}_{0.15}\right)_{3} \quad$ (labelled as CsFAMA)-mixed perovskite precursor was prepared by dissolving $1.3 \mathrm{~mol} \mathrm{~L}^{-1}$ organic cation (FAI:MABr = 0.85:0.15, by mole) and $1.4 \mathrm{~mol} \mathrm{~L}^{-1}$ mixture of metal lead salts $\left(\mathrm{PbI}_{2}: \mathrm{PbBr}_{2}=0.85: 0.15\right.$, by mole) in $0.8 \mathrm{~mL}$ mixture solvent of dimethylformamide (DMF) and dimethyl sulfoxide (DMSO) (DMF:FMSO $=4: 1$, by volume), and then $34 \mu \mathrm{L}$ CsI solution (pre-dissolved as a $2 \mathrm{~mol} \mathrm{~L}^{-1}$ stock solution in DMSO) was added to achieve the desired CsFAMA perovskite precursor solution.

\section{Preparation of the spiro-OMeTAD solution}

The Li-spiro solution was prepared by dissolving $73 \mathrm{mg}$ spiro-OMeTAD in $1 \mathrm{~mL}$ CBZ. The molar ratios of additives for spiro-OMeTAD were $0.55,3.47$ and 0.09 for Li-TFSI, $t$-BP and tris(2-(1H-pyrazol-1-yl)-4-tert-butylpyridine)-cobalt(III) tris(bis(trifluoromethylsulfonyl) imide)) (FK209), respectively.

The K-spiro solution was prepared by dissolving $20 \mathrm{mg}$ spiro-OMeTAD in $1 \mathrm{~mL}$ THF. The molar ratios of additives for spiro-OMeTAD were $x(x=0.2,0.3,0.4,0.5)$, $6.3 x$ and $0.16 x$ for K-FSI, $t$-BP and FK209, respectively.

\section{Device fabrication}

Fluorine-doped tin oxide (FTO) glass substrate was etched by a laser machine (Universal Laser Systems, VLS2.30), and followed by ultrasonic cleaning through detergent, pure water and ethyl alcohol for $20 \mathrm{~min}$, respectively. They were then dried with dry-air gas flow and then treated by plasma for $5 \mathrm{~min}$. The compact $\mathrm{SnO}_{2}$ film was achieved by a chemical bath deposition (CBD) method according to a previous report [25]. The perovskite absorber was deposited on the UV-processed $\mathrm{SnO}_{2}$ substrates (UV illuminated for $15 \mathrm{~min}$ ) by spun $25 \mu \mathrm{L}$ mixed perovskite solution at $6000 \mathrm{rpm}$ for $30 \mathrm{~s}$, and $100 \mu \mathrm{L}$ of ethyl acetate as the anti-solvent was dropped at the last $5^{\text {th }}$ second, followed by annealing at $120^{\circ} \mathrm{C}$ for $45 \mathrm{~min}$. After cooling down, Li-spiro (in CBZ) was deposited on the top of the perovskite layer directly by spincoating at $3000 \mathrm{rpm}$ for $30 \mathrm{~s}$. Alternatively, K-spiro (in THF) was deposited on top of the perovskite layer by dynamic spin-coating at $3000 \mathrm{rpm}$ for $30 \mathrm{~s}$. Finally, $80 \mathrm{~nm}$ of gold was evaporated on the top of spiro-OMeTAD as the back electrode to complete the whole devices.

\section{Characterizations}

The perovskite films were investigated using field-emission scanning electron microscope (SEM) (S-4800, Hitachi, Japan), atomic force microscope (AFM) (NX10, 
Park, Korea) and X-ray photoelectron spectroscopy (XPS) (ESCALAB 250Xi). The steady-state photoluminescence (PL) spectra were obtained using a PL microscopic spectrometer (Flex One, Zolix, China), and the time-resolved PL (TRPL) was measured at $770 \mathrm{~nm}$ using excitation with a 478-nm light pulse from Delta Flex Fluorescence Lifetime System (Horiba Scientific Com., Japan). The current density-voltage $(J-V)$ curves of these PSCs were measured using a Keithley 2400 source meter in the room environment. The light source was a solar simulator (Oriel $94023 \mathrm{~A}, 300 \mathrm{~W}$ ) matching AM 1.5 G. The intensity of the light was $100 \mathrm{~mW} \mathrm{~cm}^{-2}$ calibrated by a standard silicon reference solar cell (Oriel, VLSI standards). All the devices were tested using a black metal aperture with a defined active area of $0.16 \mathrm{~cm}^{2}$ for the small devices. Time-of-flight secondary-ion mass spectrometry (TOF-SIMS) was performed by time-of-flight secondary-ion mass spectrometer (ION-TOF GmbH 5, Germany).

The samples for X-ray photoelectron spectroscopy (XPS) measurement were prepared as follows: the $\mathrm{SnO}_{2} /$ perovskite/HTLs films were fabricated on the FTO substrate without the deposition of Au. After five days in dark, a subsequent CBZ wash was conducted to remove the spiro-OMeTAD layer. First, CBZ was dropped onto the surface of HTL to dissolve spiro-OMeTAD, then CBZ was dropped onto the exposed perovskite layer spinning at a preset rate of $3000 \mathrm{rpm}$ to remove the residual spiroOMeTAD. THF was used to wash the perovskite surface again to ensure that K-FSI was completely removed.

\section{RESULTS AND DISCUSSION}

\section{Properties of the HTLs}

Fig. 1a shows a typical normal structure of a PSC and the molecular structure of spiro-OMeTAD that is employed as the HTM. Due to the low hole mobility of spiroOMeTAD, it is necessary to introduce an efficient p-type dopant for improving its property. Li-TFSI is a conventional p-type dopant for spiro-OMeTAD and its molecular structure is shown in Fig. 1b. However, it brings about the stability issue. In order to balance the efficiency and stability simultaneously, a new dopant of K-FSI as shown in Fig. 1c was used instead. In addition, to replace the toxic solvent of CBZ, a non-halogen solvent of THF was used to dissolve spiro-OMeTAD and K-FSI. For comparison, the spiro-OMeTAD HTL prepared from the conventional dopant (Li-TFSI) with halogen solvent (CBZ) was chosen as the reference, denoted as Li-spiro. Due to the high volatilization of THF solvent, the K-FSI- doped spiro-OMeTAD (denoted as K-spiro) was deposited by a dynamic spin-coating method. Firstly, the optimal concentration of spiro-OMeTAD in THF was determined to be $20 \mathrm{mg} \mathrm{mL}^{-1}$, as shown in Fig. S1. The spiro-OMeTAD was deposited on the CsFAMA perovskite film that has large grains as shown in Fig. S2a. The SEM images of the Li-spiro and K-spiro HTLs deposited on the perovskite show a similar morphology (Fig. S2b and c). The morphology and uniformity of perovskite and HTL films were further examined by AFM, as shown in Fig. 1d, f. The perovskite film exhibits a relatively rough surface with an average surface roughness $\left(R_{\mathrm{a}}\right)$ of $15.82 \mathrm{~nm}$. After deposition of spiroOMeTAD, the films exhibit more uniform and smooth surfaces, and the corresponding roughness values of $\mathrm{Li}$ spiro and K-spiro films reduce to 3.15 and $6.84 \mathrm{~nm}$, respectively. The roughness of K-spiro film is slightly higher than that of Li-spiro film, possibly due to the thinner thickness. Different evaporation rates of different solvents would result in different packing orders of the spiro-OMeTAD. The thickness of the optimized K-spiro HTL is around $140 \mathrm{~nm}$, which is slightly thinner than that of Li-spiro HTL, confirmed by the cross-sectional SEM images (Fig. S2d and e).

In order to evaluate the hole transporting property of these two spiro-OMeTAD films, the hole mobility was measured with a hole-only device of indium tin oxidecoated glass substrate (ITO)/poly(3,4-ethylenedioxythiophene)-poly(styrenesulfonate) (PEDOT:PSS) (40 nm) /spiro-OMeTAD/ $/ \mathrm{MoO}_{3}(10 \mathrm{~nm}) / \mathrm{Ag}$ using the space charge limited current (SCLC) method as shown in Fig. 2a. The hole mobility can be calculated from Equation (1):

$J=\frac{9}{8} \varepsilon_{0} \varepsilon_{1} \mu \frac{V^{2}}{d^{3}}$,

where $\mu$ is the hole mobility, $\varepsilon_{0}$ is permittivity of free space $\left(\varepsilon_{0}=8.8542 \times 10^{-14} \mathrm{~F} \mathrm{~cm}^{-1}\right)$, $\varepsilon_{\mathrm{r}}$ is the dielectric constant of spiro-OMeTAD $\left(\varepsilon_{\mathrm{r}}=3\right)$, and $d$ is the film thickness. To maintain consistency with the HTL for photovoltaic devices, the composition of the HTLs contain spiro-OMeTAD, $t$-BP, FK209 and either Li-TFSI or K-FSI for the hole mobility measurements. By fitting the $J-V$ curves, the hole mobility of pristine spiro-OMeTAD without any additives is calculated to be $4.71 \times 10^{-4} \mathrm{~cm}^{2} \mathrm{~V}^{-1} \mathrm{~s}^{-1}$, which is consistent with the previous report [21]. With the doping of Li-TFSI, the hole mobility significantly increases to $2.61 \times 10^{-3} \mathrm{~cm}^{2} \mathrm{~V}^{-1} \mathrm{~s}^{-1}$, exhibiting one order of magnitude improvement compared with the undoped spiro-OMeTAD. When Li-TFSI 

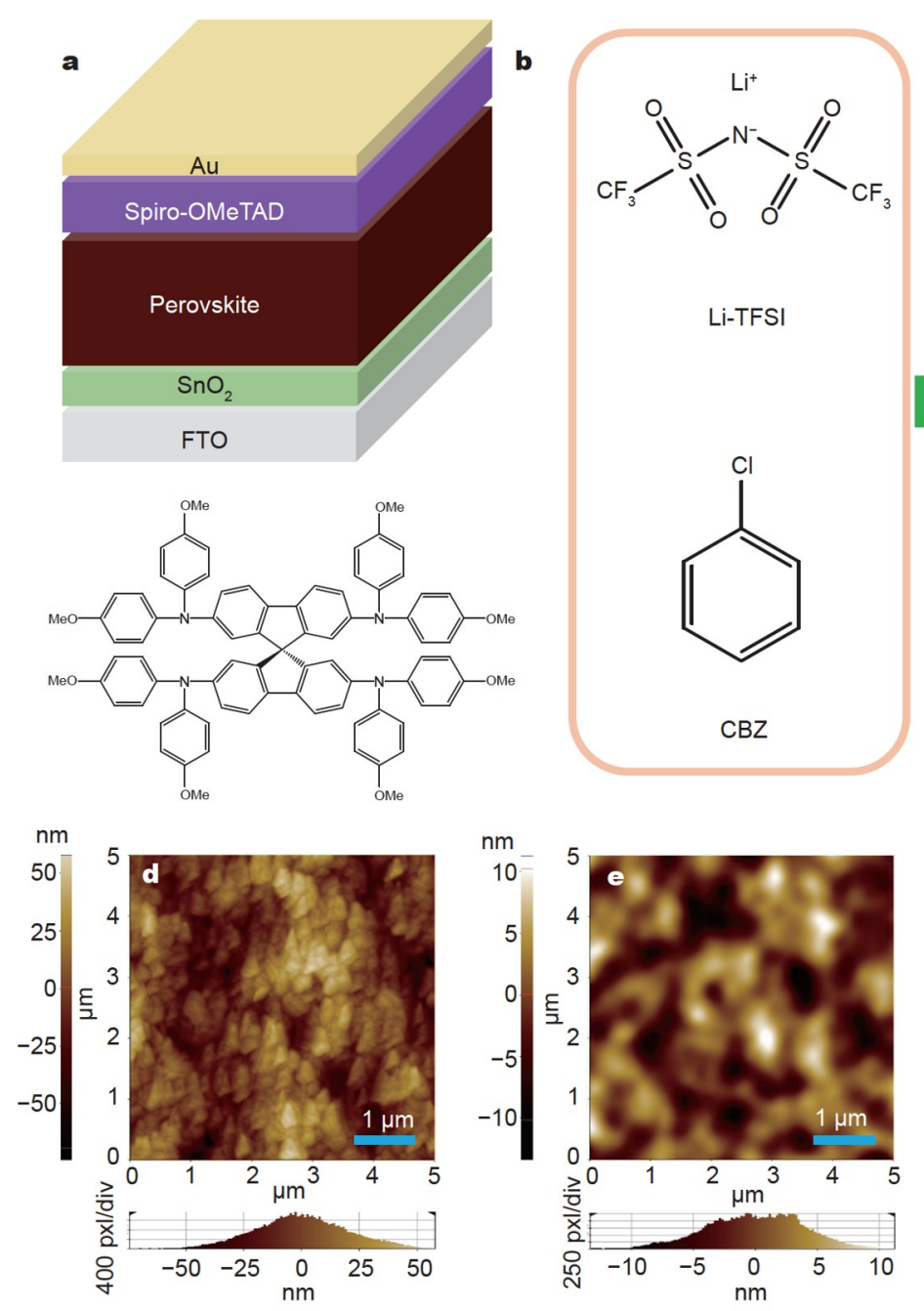
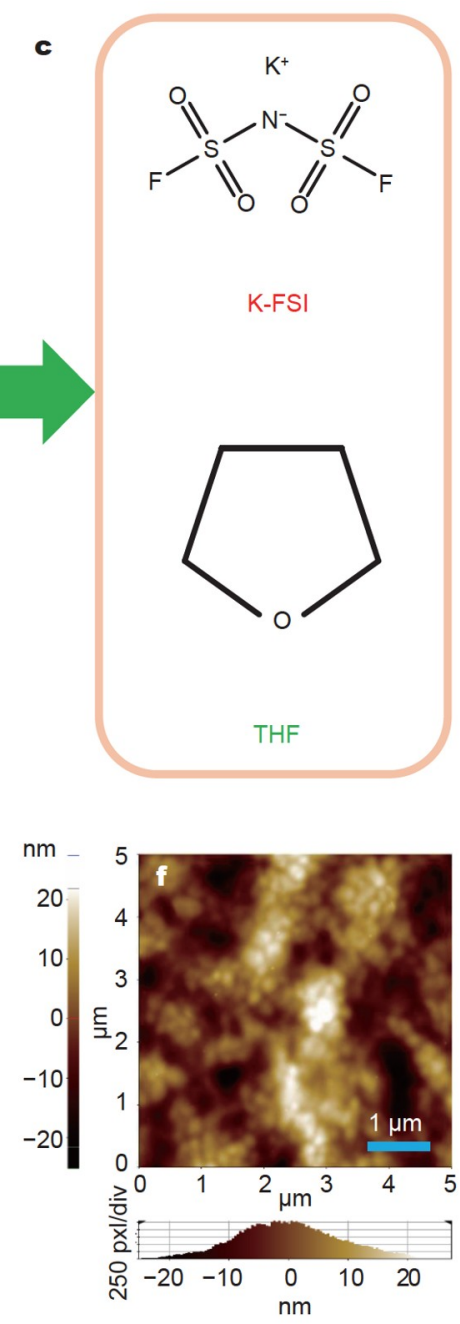

Figure 1 (a) A typical normal structure of planar PSCs, and the molecular structure of spiro-OMeTAD. (b, c) The schematic of the different dopants and solvents for HTL. (d-f) AFM images of the perovskite film, perovskite/Li-spiro, and perovskite/K-spiro, respectively.
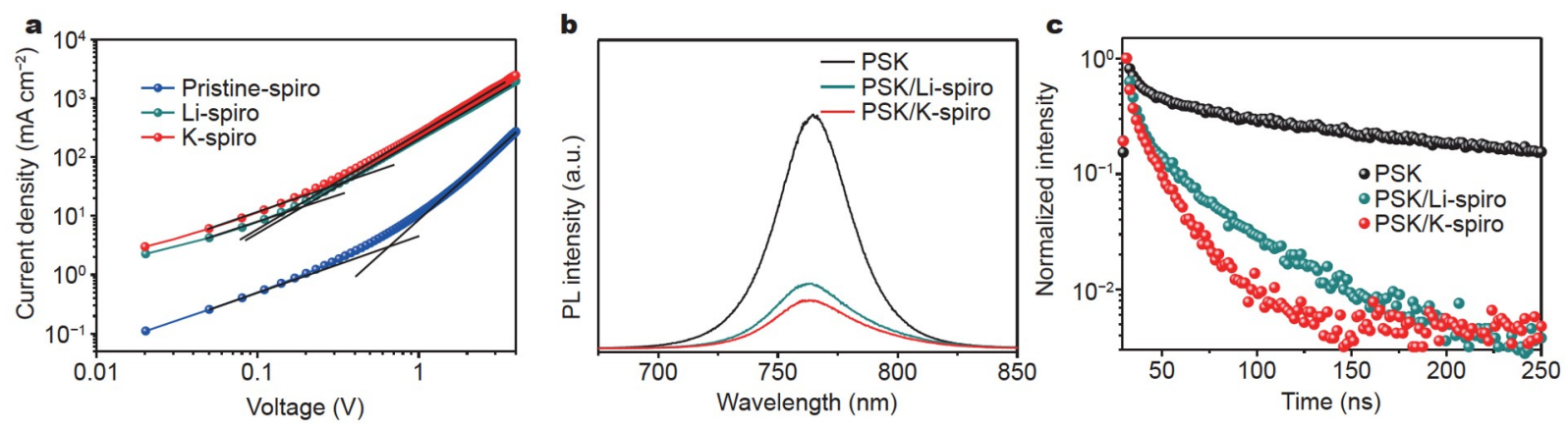

Figure 2 (a) $J-V$ characteristics of hole-only devices of pristine spiro-OMeTAD, Li-spiro and K-spiro films. (b) Steady-state PL and (c) TRPL measurements of perovskite (PSK), perovskite/Li-spiro and perovskite/K-spiro films.

is substituted by K-FSI, the hole mobility further increases to $3.62 \times 10^{-3} \mathrm{~cm}^{2} \mathrm{~V}^{-1} \mathrm{~s}^{-1}$, about $40 \%$ higher than that of Li-TFSI. Li-TFSI promotes the generation of holes through forming a radical cation (spiro-OMeTAD ${ }^{\circ}{ }^{-}$ 
TFSI $^{-}$), which is weakly bound by the extensively delocalized charge on the TFSI anion [13]. This anionic charge is highly delocalized on the disulfonylimide moiety, which is the main component for both TFSI and FSI anions [26]. Thus, we speculate that K-FSI can also result in the effective generation of mobile holes on the organic matrix by the formation of spiro-OMeTAD ${ }^{\circ+} \mathrm{FSI}^{-}$.

Mott-Schottky analysis was used to further explore the carrier concentration of different HTLs (Fig. S3). The carrier density of K-spiro was calculated to be $1.42 \times 10^{20} \mathrm{~cm}^{-3}$, which is slightly higher than that of Lispiro $\left(1.27 \times 10^{20} \mathrm{~cm}^{-3}\right)$, reflecting an increase in the hole concentration. Meanwhile, the HOMO level of K-spiro is calculated to be $-5.226 \mathrm{eV}$ from Mott-Schottky analysis, lower than that of Li-spiro $(-5.212 \mathrm{eV})$, obtaining a better energy level match between the HTL and perovskite layer.

The conductance properties of different HTLs were characterized by $J-V$ curves. The device structure is FTO/ spiro-OMeTAD/Au. It can be found that the slope of the $J-V$ curve follows a trend of $\mathrm{K}$-spiro $>\mathrm{Li}$-spiro $>$ pristine spiro (Fig. S4), indicating that the K-spiro exhibits the best conductance property.

PL spectra were further employed to evaluate the hole extraction properties for these two spiro-OMeTAD films. Fig. $2 b$ shows the steady-state PL spectra for the neat perovskite film and perovskite films covered with the $\mathrm{Li}$ spiro and K-spiro films, respectively. The pristine perovskite film presents a strong PL peak at $765 \mathrm{~nm}$, which is diminished by attaching Li-spiro film obviously. The intensity can be further diminished by the K-spiro film, reflecting faster charge separation and extraction and less charge recombination at the interface compared with the Li-spiro. This consequence is subsequently evident from the TRPL measurements, as depicted in Fig. 2c. The TRPL spectra are fitted on the basis of a biexponential PL decay process [27], and the fitted parameters are listed in Table S1. The fast decay component $\left(\tau_{1}\right)$ mainly represents the non-radiative recombination induced by trap states at grain boundaries and surfaces. The $\tau_{1}$ of the perovskite/K-spiro sample $(1.6 \mathrm{~ns})$ and the average PL decay time $(10.1 \mathrm{~ns})$ are shorter than those of the perovskite/Li-spiro sample (3.0 and $21.1 \mathrm{ns,} \mathrm{respectively),}$ demonstrating more rapid and efficient hole transfer into spiro-OMeTAD with less non-radiative recombination due to the decreased interfacial trap state and defect passivation. The better charge extraction ability of the Kspiro should have benefited from the effective interface passivation by $\mathrm{K}$ ions and the enhanced conductivity and hole mobility of the K-spiro. Therefore, an increased open-circuit voltage $\left(V_{\mathrm{OC}}\right)$ could be expected in the PSCs.

\section{Photovoltaic performance}

Fig. 3a shows the $J-V$ curves of the champion Li-spiro and $\mathrm{K}$-spiro devices, and the corresponding photovoltaic characteristics are listed in Table 1 . Under the optimal doping concentration of $40 \% \mathrm{~K}$-FSI, the K-spiro device exhibits the highest PCE of $20.94 \%$ under RS and $21.02 \%$ under FS, with a $V_{\mathrm{OC}}$ of $1.159 \mathrm{~V}$, a short-circuit current density $\left(J_{\mathrm{SC}}\right)$ of $22.98 \mathrm{~mA} \mathrm{~cm}^{-2}$ and a fill factor $(\mathrm{FF})$ of 0.79. As a contrast, the champion PSC device utilizing LiTFSI shows a relatively low PCE of $19.75 \%$, with a $V_{\text {OC }}$ of $1.128 \mathrm{~V}$, a $J_{\mathrm{SC}}$ of $22.62 \mathrm{~mA} \mathrm{~cm}^{-2}$ and an FF of 0.77 . Obviously, the improvement of these photovoltaic characteristics is due to the substitution of the Li-spiro with the K-spiro. We can observe an increase of $V_{\mathrm{OC}}$ from 1.128 to $1.159 \mathrm{~V}$, which is consistent with the speculation according to the PL measurement. External quantum efficiency (EQE) characterization was conducted to evaluate the increase in $J_{\mathrm{SC}}$. A slightly higher EQE of the $\mathrm{K}$-spiro device than that of the control device occurs in the spectra range from 630 to $750 \mathrm{~nm}$ (Fig. 3b). Thus, the integrated $J_{\mathrm{SC}}$ is calculated to be $22.26 \mathrm{~mA} \mathrm{~cm}^{-2}$ for the $\mathrm{K}$ spiro device, higher than $21.84 \mathrm{~mA} \mathrm{~cm}^{-2}$ of the Li-spiro device, matching well with the $J_{\mathrm{SC}}$ obtained from the $J-V$ curves. Moreover, Fig. $3 \mathrm{c}$ records the stabilized power output (SPO) measurement of above devices, the K-spiro device achieves a stabilized PCE of $20.00 \% \pm 0.10 \%$ in $120 \mathrm{~s}$ at the maximum power point (MPP) at a bias of $0.98 \mathrm{~V}$, whereas the PCE of Li-spiro device decreases slowly from $17.56 \%$ to $16.97 \%$ during the same test duration at a bias of $0.93 \mathrm{~V}$, obtaining an average PCE of $17.21 \%$. This result indicates superior photostability of the K-spiro device compared with the Li-spiro one. Simultaneously, to further examine the reproducibility of these enhancements, 12 PSCs based on the Li-spiro and K-spiro HTLs were fabricated and tested under AM 1.5 G illumination with RS and FS (Fig. 3d). Statistical results for the photovoltaic parameters with respect to the HTLs are displayed in Fig. S5, showing an overall higher average PCE for the K-spiro device $(20.31 \% \pm 0.31 \%)$ than that of the Li-spiro device $(19.30 \% \pm 0.27 \%)$. The above results clearly show the benefit of K-FSI as the HTL dopant.

\section{Hysteresis study}

Hysteresis is a common issue in normal planar PSCs [28], which has been proved to have an impact on the device performance and stability without a unified solution. The main causes of this phenomenon are regarded as ion migration and charge trapping related to defects [28-30]. Usually, hysteresis index (HI) is employed to quantify this 

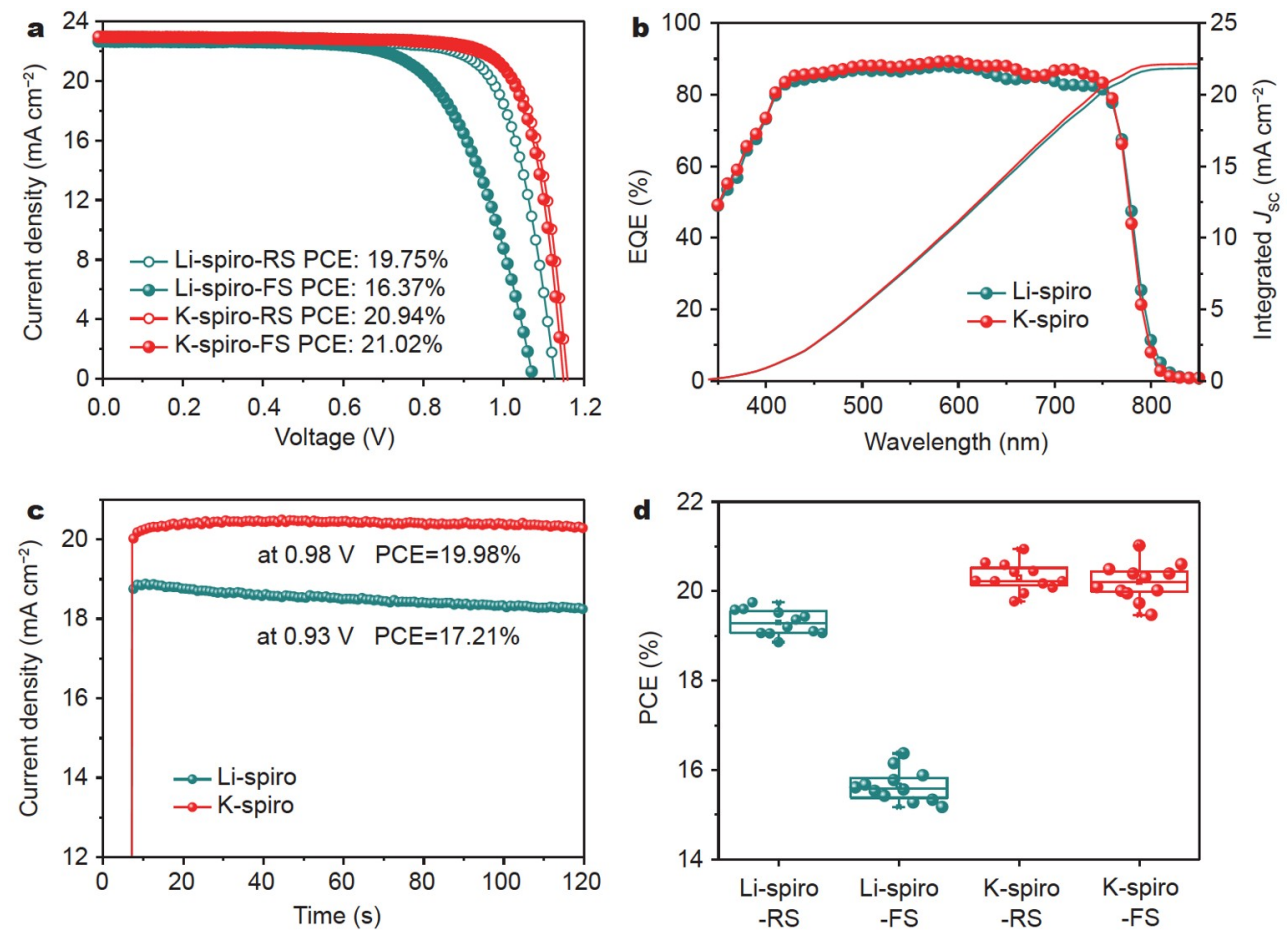

Figure 3 (a) The $J-V$ curves of the champion Li-spiro and K-spiro devices tested under 1 Sun AM 1.5G. (b) The corresponding EQE spectra of these devices. (c) Steady-state output of photocurrent density measured at the MPP and illuminated under 1 Sun AM 1.5G. (d) Statistical distribution of the PCEs for the Li-spiro and K-spiro devices under RS and FS.

Table 1 Photovoltaic characteristics of PSCs prepared with different HTLs

\begin{tabular}{ccccccc}
\hline HTL & Sweep & $V_{\text {OC }}(\mathrm{V})$ & $\begin{array}{c}J_{\text {SC }} \\
\left(\mathrm{mA} \mathrm{cm}^{-2}\right)\end{array}$ & FF & PCE (\%) & HI \\
\hline \multirow{2}{*}{ Li-spiro } & RS & 1.128 & 22.62 & 0.77 & 19.75 & 0.171 \\
& FS & 1.073 & 22.64 & 0.67 & 16.37 & \\
& KS & 1.159 & 22.98 & 0.79 & 20.94 & 0.004 \\
& FS & 1.149 & 22.93 & 0.80 & 21.02 & \\
\hline
\end{tabular}

discrepancy of photovoltaic performance by the following equation: $\mathrm{HI}=\left(\mathrm{PCE}_{\mathrm{RS}}-\mathrm{PCE}_{\mathrm{FS}}\right) / \mathrm{PCE}_{\mathrm{RS}}$. In the literature, $\mathrm{Li}, \mathrm{Na}, \mathrm{Mg}, \mathrm{Ca}, \mathrm{Ag}$, and $\mathrm{Zn}$-TFSI have been used as dopants, but all have a high HI (Table S2). However, it is found that the PSCs based on the K-spiro exhibit a negligible hysteresis with the $\mathrm{HI}$ of only 0.004 , whereas the PSCs based on the Li-TFSI show obvious hysteresis with the HI of 0.171 (Fig. 3d). We proceeded to investigate which component of K-FSI was dominant for hysteresis elimination by fabricating devices with different compositions of dopants for HTLs. The PSCs with Li$\mathrm{TFSI} / t$-BP and Li-FSI/t-BP were first characterized to probe the impact of TFSI and FSI anions. As presented in Table S3, the Li-FSI/t-BP device achieves PCEs of $19.06 \%$ and $14.58 \%$ under RS and FS, respectively, with no suppression of hysteresis compared with Li-TFSI/t-BP device. Thus the replacement of TFSI by FSI anion has only a slight effect on hysteresis. Meanwhile, we also find that cobalt salt is not the decisive factor for hysteresis elimination. Device containing only cobalt salts and $t$-BP presents PCEs of $17.98 \%$ and $15.47 \%$ under RS and FS with an $\mathrm{HI}$ of 0.140 ; the decrease of $\mathrm{HI}$ could be attributed to the absence of lithium in the device. It has been proven that suppressing the migration of lithium from HTL to ETL could result in the reduced hysteresis of the PSCs $[17,31]$. In the absence of cobalt salt, devices involving K-FSI/t-BP obtain PCEs of $19.88 \%$ and $19.45 \%$ under RS and FS, higher than that of $19.16 \%$ and $14.57 \%$ for the devices using Li-TFSI/ $t$-BP (Table S3). The tendency of HI between K-FSI and Li-TFSI was similar with or without cobalt salt. Hence, we can conclude that the negligible hysteresis results from the inclusion of $\mathrm{K}$ cation in HTL, while cobalt salt plays a role in improving the overall PCEs by helping oxidization of spiro-OMeTAD [32]. It is noteworthy that although with excessive $\mathrm{K}$ doping, the PCE decreases, all the devices with different $\mathrm{K}$ doping concentrations display a negligible hysteresis under different concentrations of K-FSI (Table S4). To in- 
vestigate the intrinsic reason of the suppression of hysteresis, TOF-SIMS measurement was conducted to detect the distribution of several key elements in the whole device. It is important to note that the thickness of each layer is not in direct proportion to the sputter time because different layers behave different sputtering rates, which is related to the bond strength of materials. Fig. 4a shows the TOF-SIMS analysis of the Li-spiro device, which reveals that $\mathrm{Li}$ is located in the spiro-OMeTAD layer. Besides, there is also a comparable amount of $\mathrm{Li}$ at the perovskite/ $\mathrm{SnO}_{2}$ interface, while only a small amount of Li remains in the perovskite layer, which does not follow the Fick's law of diffusion. This suggests that the $\mathrm{Li}^{+}$ions in HTL tend to migrate through the perovskite layer and accumulate at the perovskite/ $\mathrm{SnO}_{2}$ interface on account of the smaller radius of $\mathrm{Li}$ than other constituent elements of PSCs and a higher affinity of $\mathrm{Li}^{+}$ions to $\mathrm{SnO}_{2}$ than to perovskite [29]. However, the distribution of $\mathrm{K}$ shows a different tendency from $\mathrm{Li}$, as depicted in Fig. $4 \mathrm{~b}$. More $\mathrm{K}^{+}$ions remain in the spiro-OMeTAD layer than at the perovskite/ $\mathrm{SnO}_{2}$ interface as $\mathrm{K}^{+}$is much larger than $\mathrm{Li}^{+}$.

To confirm that potassium exists in some form at the $\mathrm{HTL} /$ perovskite interface, we carried out XPS to probe the surface electronic properties of the perovskite films after removal of the top HTLs. The devices were firstly fabricated as usual without the subsequent deposition of $\mathrm{Au}$ and kept in dark for five days. Then the HTLs were removed with CBZ and the HTL doped by K-FSI was additionally removed with THF. It is long enough for $\mathrm{Li}^{+}$ and $\mathrm{K}^{+}$to diffuse from HTL to perovskite or even $\mathrm{SnO}_{2}$ layer in five days. After the removal of Li-spiro, no obvious lithium signal is detected on the surface of the perovskite film as seen in the XPS spectrum (Fig. 4c), which indicates that $\mathrm{Li}^{+}$does not tend to interact with the elements in perovskite at the HTL/perovskite interface. However, an apparent peak of $\mathrm{K} 2 \mathrm{p}$ orbital appears in the sample with K-FSI after treatment with CBZ and THF, as shown in Fig. 4d. This result suggests that $\mathrm{K}^{+}$ions could bond with perovskite rather than simply being coated on the perovskite surface. Therefore, it is reasonable to believe that the $\mathrm{K}^{+}$at the $\mathrm{HTL} /$ perovskite interface leads to the enhancement of voltaic performance and hysteresis elimination, which could be explained from two respects. On the one hand, the electron clouds around $\mathrm{Br}$ can be substantially diminished when the HTL/perovskite interface is modified with $\mathrm{K}^{+}$, weakening dangling bonds on the perovskite surface [33]. We believe that the $\mathrm{K}-\mathrm{Br}$ and
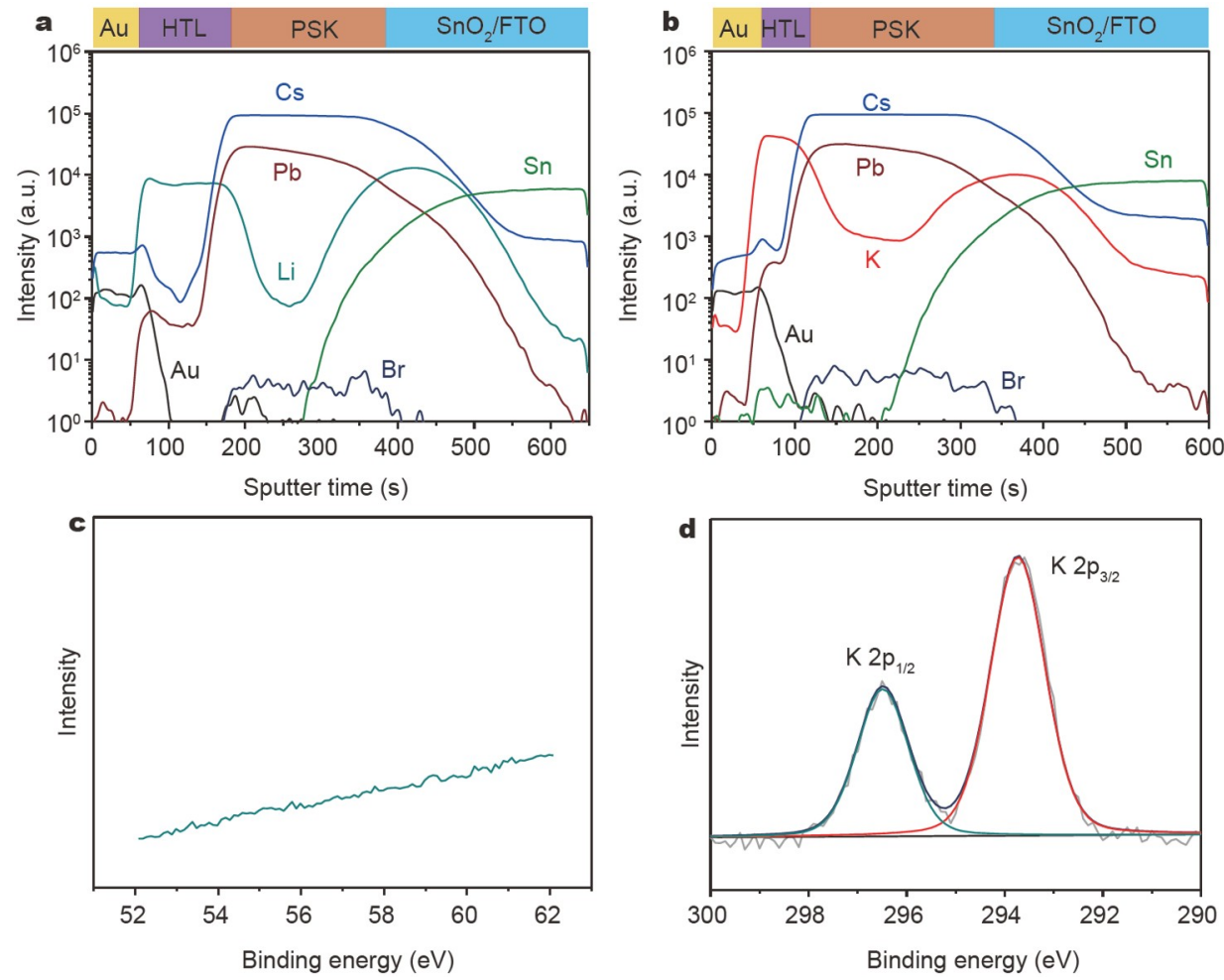

Figure 4 The TOF-SIMS depth profiles of (a) Li-spiro device and (b) K-spiro device. The XPS spectra of perovskite films after removal of (c) Lispiro and (d) K-spiro deposited five days ago. 
$\mathrm{K}-\mathrm{I}$ bonds will preferentially form due to the strong ionic interaction between $\mathrm{K}^{+}$and halide ions. The interaction between $\mathrm{K}$ and $\mathrm{Br} / \mathrm{I}$ passivates the halide defects at the interface to reduce the non-radiative recombination and ion migration in perovskite films [34-36]. On the other hand, Frenkel defect is also be supposed to cause the hysteresis effect, which occurs with the accumulation of excess electrons at the cathode under illumination. $\mathrm{K}^{+}$ ions (radius of $1.38 \AA$ ) can be well stabilized in the octahedral site to protect the Frenkel defect due to the appropriate radius ratio while $\mathrm{Li}^{+}$and $\mathrm{Cs}^{+}$ions fail to occupy the interstitial sites stably in octahedron [28]. This is also the reason why hysteresis can be largely suppressed by $\mathrm{K}^{+}$passivation whereas devices with $\mathrm{Li}$ or Cs still exhibit severe hysteresis.

To further investigate the charge recombination in PSCs, we measured the $V_{\mathrm{OC}}$ of the Li-spiro and K-spiro devices under various light intensities, as shown in Fig. S6. It is known that the deviation of the slope from $k T / q$ (where $k$ is the Boltzmann constant, $T$ represents the absolute temperature, and $q$ denotes the elementary charge) reflects a trap-assisted recombination in PSCs [37]. The K-spiro device has a slope of $1.52 \mathrm{kT} / \mathrm{q}$, which is lower than that of the Li-spiro device $(2.22 \mathrm{kT} / \mathrm{q})$. It indicates a suppressed trap-assisted recombination at the interface after $\mathrm{K}^{+}$passivation, revealing that the $\mathrm{K}$-spiro provides a better interface contact for the PSCs. This is also confirmed by the characterization of electrochemical impedance spectroscopy (EIS), which is utilized to eval-

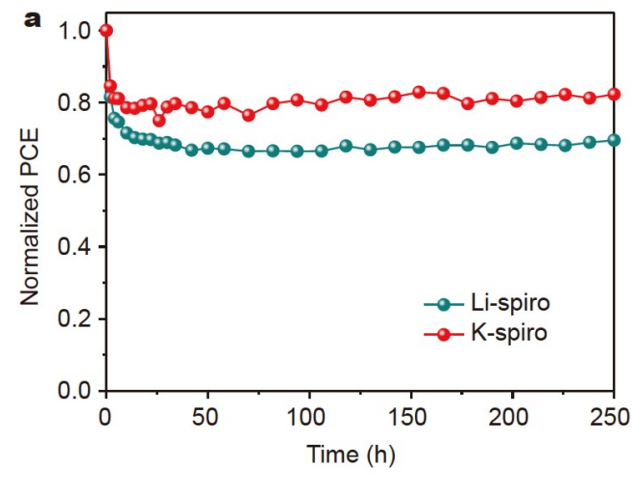

c

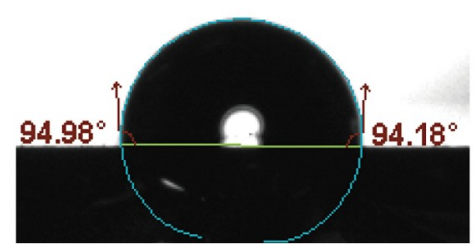

uate the charge transport resistance $\left(R_{\mathrm{ct}}\right)$ and recombination resistance $\left(R_{\text {rec }}\right)[38,39]$. As presented in Fig. S7, EIS was measured at a bias of $1.0 \mathrm{~V}$ under simulated AM 1.5 illumination, two semicircle regions can be observed in the Nyquist plot where the high frequency component correlates with the charge transfer process and the low frequency region stands for charge recombination at the interface. The fitted $R_{\mathrm{ct}}$ of the K-spiro device is lower than that of the Li-spiro device according to the first arc located on high frequency, while the second arc indicates a larger $R_{\text {rec }}$ of the K-spiro device than that of the Li-spiro device, demonstrating a reduced charge recombination and hence a higher $V_{\mathrm{OC}}$. Therefore, the above results reveal that the K-spiro reduces defects and traps at the perovskite/HTL interface and provides a better interface contact for the PSCs.

\section{Stability}

The stability of devices based on different dopants was also characterized against light illumination and humid environment. It is observed that the K-spiro device shows a good photo-stability. As shown in Fig. 5a, the encapsulated K-spiro device maintains about $82 \%$ of the initial PCE under continuous 0.6 sun illumination during $250 \mathrm{~h}$, while the PCE of the Li-spiro device reduces to $69 \%$ compared with the initial value. The improvement of photo-stability is mainly attributed to the $\mathrm{K}^{+}$passivation of interfacial trapping defects and the suppression of ion migration, which is prone to being triggered under the

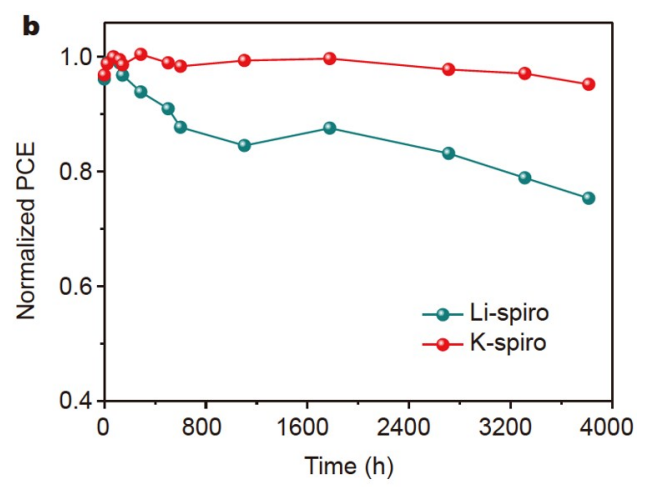

d

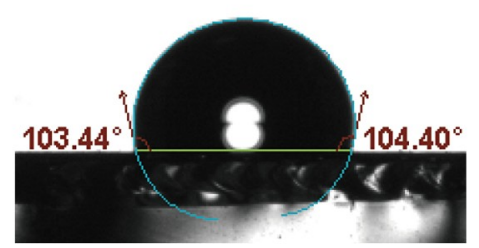

Figure 5 (a) The long-term photo-stability of devices under continuous 0.6 sun light illumination in air with encapsulation. (b) Stability test of the devices without encapsulation aged in air (relative humidity $25 \%$, at $25^{\circ} \mathrm{C}$ ). Contact angle measurements of (c) Li-spiro and (d) K-spiro films. 
light illumination. On the contrary, the migration and accumulation of $\mathrm{Li}^{+}$at the perovskite/ETL interface have been regarded to be detrimental to the photo-stability of the devices. Moreover, Fig. 5b displays the normalized PCE of unencapsulated devices with respect to the dopant under a condition of $25 \%$ relative humidity (RH) after 140 days. The K-spiro device exhibits a superior humidity stability with only $3 \%$ decrease of the PCE, while the Lispiro device shows $22 \%$ drop in the same condition. In addition, we measured the water contact angles of the different spiro-OMeTAD films (Fig. $5 \mathrm{c}$ and d). It can be seen that the K-spiro displays a larger contact angle $\left(104^{\circ}\right)$ than the Li-spiro $\left(94^{\circ}\right)$, resulting in the enhanced hydrophobicity of the K-spiro. Thus, K-FSI is a more reliable dopant for spiro-OMeTAD to promote the operational long-term stability of PSCs.

\section{CONCLUSIONS}

In summary, we develop an innovative p-dopant for spiro-OMeTAD HTL to improve the performance and stability of PSCs by substituting the traditional additive of Li-TFSI with K-FSI. The K-doped HTL attains a higher conductivity and hole mobility, and provides significantly enhanced hole extraction as well as reduced charge recombination for the PSCs. As a consequence, the device with K-FSI achieves an impressive PCE of $20.94 \%$ for RS and $21.02 \%$ for FS based on an increased $V_{\mathrm{OC}}$. The most remarkable thing is that the notorious hysteresis effect is completely eliminated. Characterizations suggest that $\mathrm{K}^{+}$ ions migrate from the HTL to perovskite to form $\mathrm{K}-\mathrm{Br} / \mathrm{I}$, thus passivating the surface defects and mitigating halide migration at the HTL/perovskite interface, which results in the high-performance and hysteresis-free PSCs. Meanwhile, the stability of devices against light illumination and ambient environment are both enhanced by the incorporation of $\mathrm{K}$. In addition, THF successfully replaces the toxic $\mathrm{CBZ}$ as the solvent for spiro-OMeTAD, which is more conducive to the industrial production of PSC devices. This work provides a new way to fabricate efficient, stable and hysteresis-free PSCs.

\section{Received 3 March 2021; accepted 10 May 2021;} published online 6 July 2021

1 Park NG. Perovskite solar cell: Research direction for next 10 years. ACS Energy Lett, 2019, 4: 2983-2985

2 https://www.nrel.gov/pv/cell-efficiency.html

3 Kojima A, Teshima K, Shirai Y, et al. Organometal halide perovskites as visible-light sensitizers for photovoltaic cells. J Am Chem Soc, 2009, 131: 6050-6051

4 Green MA, Ho-Baillie A, Snaith HJ. The emergence of perovskite solar cells. Nat Photon, 2014, 8: 506-514
5 Xing G, Mathews N, Sun S, et al. Long-range balanced electronand hole-transport lengths in organic-inorganic $\mathrm{CH}_{3} \mathrm{NH}_{3} \mathrm{PbI}_{3}$. Science, 2013, 342: 344-347

6 Stranks SD, Eperon GE, Grancini G, et al. Electron-hole diffusion lengths exceeding 1 micrometer in an organometal trihalide perovskite absorber. Science, 2013, 342: 341-344

7 Jiang Q, Zhao Y, Zhang X, et al. Surface passivation of perovskite film for efficient solar cells. Nat Photonics, 2019, 13: 460-466

8 Saparov B, Mitzi DB. Organic-inorganic perovskites: Structural versatility for functional materials design. Chem Rev, 2016, 116: 4558-4596

9 Shao JY, Yu B, Wang YD, et al. In-situ electropolymerized polyamines as dopant-free hole-transporting materials for efficient and stable inverted perovskite solar cells. ACS Appl Energy Mater, 2020, 3: 5058-5066

10 Zhang L, Wu J, Li D, et al. Ladder-like conjugated polymers used as hole-transporting materials for high-efficiency perovskite solar cells. J Mater Chem A, 2019, 7: 14473-14477

11 Li D, Shao JY, Li Y, et al. New hole transporting materials for planar perovskite solar cells. Chem Commun, 2018, 54: 1651-1654

12 Burschka J, Dualeh A, Kessler F, et al. Tris(2-(1H-pyrazol-1-yl) pyridine)cobalt(III) as p-type dopant for organic semiconductors and its application in highly efficient solid-state dye-sensitized solar cells. J Am Chem Soc, 2011, 133: 18042-18045

13 Abate A, Leijtens T, Pathak S, et al. Lithium salts as "redox active" p-type dopants for organic semiconductors and their impact in solid-state dye-sensitized solar cells. Phys Chem Chem Phys, 2013, 15: 2572-2579

14 Habisreutinger SN, Noel NK, Snaith HJ, et al. Investigating the role of 4-tert butylpyridine in perovskite solar cells. Adv Energy Mater, 2017, 7: 1601079

15 Wang S, Sina M, Parikh P, et al. Role of 4-tert-butylpyridine as a hole transport layer morphological controller in perovskite solar cells. Nano Lett, 2016, 16: 5594-5600

16 Jena AK, Ikegami M, Miyasaka T. Severe morphological deformation of spiro-OMeTAD in $\left(\mathrm{CH}_{3} \mathrm{NH}_{3}\right) \mathrm{PbI}_{3}$ solar cells at high temperature. ACS Energy Lett, 2017, 2: 1760-1761

17 Liu Y, Hu Y, Zhang X, et al. Inhibited aggregation of lithium salt in spiro-OMeTAD toward highly efficient perovskite solar cells. Nano Energy, 2020, 70: 104483

18 Hawash Z, Ono LK, Raga SR, et al. Air-exposure induced dopant redistribution and energy level shifts in spin-coated spiro-MEoTAD films. Chem Mater, 2015, 27: 562-569

19 Wang S, Huang Z, Wang X, et al. Unveiling the role of $t$ BP-LiTFSI complexes in perovskite solar cells. J Am Chem Soc, 2018, 140: $16720-16730$

20 Seo JY, Kim HS, Akin S, et al. Novel p-dopant toward highly efficient and stable perovskite solar cells. Energy Environ Sci, 2018, 11: 2985-2992

21 Li M, Wang ZK, Yang YG, et al. Copper salts doped spiro-ometad for high-performance perovskite solar cells. Adv Energy Mater, 2016, 6: 1601156

22 Pham ND, Shang J, Yang Y, et al. Alkaline-earth bis(trifluoromethanesulfonimide) additives for efficient and stable perovskite solar cells. Nano Energy, 2020, 69: 104412

23 Zhang J, Zhang T, Jiang L, et al. 4-tert-Butylpyridine free hole transport materials for efficient perovskite solar cells: A new strategy to enhance the environmental and thermal stability. ACS Energy Lett, 2018, 3: 1677-1682

24 Caliò L, Salado M, Kazim S, et al. A generic route of hydrophobic 
doping in hole transporting material to increase longevity of perovskite solar cells. Joule, 2018, 2: 1800-1815

$25 \mathrm{Bu}$ T, Liu X, Zhou Y, et al. A novel quadruple-cation absorber for universal hysteresis elimination for high efficiency and stable perovskite solar cells. Energy Environ Sci, 2017, 10: 2509-2515

26 Djellab H, Armand M, Delabouglise D. Stabilization of the conductivity of poly(3-methylthiophene) by triflimide anions. Synth Met, 1995, 74: 223-226

27 Shi D, Adinolfi V, Comin R, et al. Low trap-state density and long carrier diffusion in organolead trihalide perovskite single crystals. Science, 2015, 347: 519-522

28 Kang DH, Park NG. On the current-voltage hysteresis in perovskite solar cells: Dependence on perovskite composition and methods to remove hysteresis. Adv Mater, 2019, 31: 1805214

29 Li Z, Xiao C, Yang Y, et al. Extrinsic ion migration in perovskite solar cells. Energy Environ Sci, 2017, 10: 1234-1242

$30 \mathrm{Yu} \mathrm{H}, \mathrm{Lu} \mathrm{H}$, Xie F, et al. Native defect-induced hysteresis behavior in organolead iodide perovskite solar cells. Adv Funct Mater, 2016, 26: 1411-1419

31 Jiang LL, Wang ZK, Li M, et al. Flower-like $\mathrm{MoS}_{2}$ nanocrystals: a powerful sorbent of $\mathrm{Li}^{+}$in the Spiro-OMeTAD layer for highly efficient and stable perovskite solar cells. J Mater Chem A, 2019, 7: 3655-3663

32 Burschka J, Kessler F, Nazeeruddin MK, et al. Co(iii) complexes as p-dopants in solid-state dye-sensitized solar cells. Chem Mater, 2013, 25: 2986-2990

33 Shen Y, Shen K-, Li Y-, et al. Interfacial potassium-guided grain growth for efficient deep-blue perovskite light-emitting diodes. Adv Funct Mater, 2021, 31: 2006736

34 Abdi-Jalebi M, Andaji-Garmaroudi Z, Cacovich S, et al. Maximizing and stabilizing luminescence from halide perovskites with potassium passivation. Nature, 2018, 555: 497-501

35 Zheng $\mathrm{F}$, Chen $\mathrm{W}, \mathrm{Bu} \mathrm{T}$, et al. Triggering the passivation effect of potassium doping in mixed-cation mixed-halide perovskite by light illumination. Adv Energy Mater, 2019, 9: 1901016

$36 \mathrm{Bu} \mathrm{T}$, Li J, Zheng F, et al. Universal passivation strategy to slot-die printed $\mathrm{SnO}_{2}$ for hysteresis-free efficient flexible perovskite solar module. Nat Commun, 2018, 9: 4609

37 Wetzelaer GJAH, Scheepers M, Sempere AM, et al. Trap-assisted non-radiative recombination in organic-inorganic perovskite solar cells. Adv Mater, 2015, 27: 1837-1841

38 Yang M, Guo R, Kadel K, et al. Improved charge transport of Nbdoped $\mathrm{TiO}_{2}$ nanorods in methylammonium lead iodide bromide perovskite solar cells. J Mater Chem A, 2014, 2: 19616-19622

39 Pascoe AR, Duffy NW, Scully AD, et al. Insights into planar $\mathrm{CH}_{3} \mathrm{NH}_{3} \mathrm{PbI}_{3}$ perovskite solar cells using impedance spectroscopy. J Phys Chem C, 2015, 119: 4444-4453

40 Bang SM, Shin SS, Jeon NJ, et al. Defect-tolerant sodium-based dopant in charge transport layers for highly efficient and stable perovskite solar cells. ACS Energy Lett, 2020, 5: 1198-1205

$41 \mathrm{Xu} \mathrm{B}$, Huang J, Ågren $\mathrm{H}$, et al. AgTFSI as p-Type dopant for efficient and stable solid-state dye-sensitized and perovskite solar cells. ChemSusChem, 2014, 7: 3252-3256

Acknowledgements This work was financially supported by the National Key Research and Development Plan (2019YFE0107200 and 2017YFE0131900), the National Natural Science Foundation of China (21875178 and 91963209), and Foshan Xianhu Laboratory of the Advanced Energy Science and Technology Guangdong Laboratory (XHD2020-001 and XHT2020-005). The Analytical and Testing Centre of Wuhan University of Technology and Hubei Key Laboratory of Low Dimensional Optoelectronic Material and Devices, Hubei University of Arts and Science is acknowledged for the XPS, XRD and SEM characterizations.

Author contributions Huang $\mathrm{F}$ and $\mathrm{Bu} \mathrm{T}$ proposed and supervised the project. Lin $\mathrm{Z}$ conducted most of the experiments and analyzed the data as well as wrote the manuscript. Li J contributed to the experimental scheme and materials selection. Li $\mathrm{H}$ and Pan J performed the PL measurement. Mo Y and Wang C performed the stability test. Zhang XL, Zhong J, and Cheng YB revised the manuscript. All the authors contributed to the general discussion.

Conflict of interest The authors declare that they have no conflict of interest.

Supplementary information

Supporting data are available in the online version of the paper.

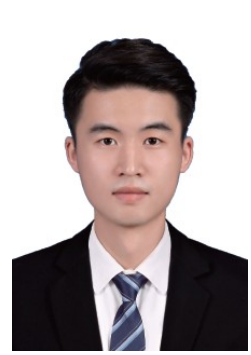

Zhipeng Lin received his BS degree from Wuhan University of Technology (WUT) in 2019. He is currently a master degree candidate at Wuhan University of Technology. His current research interest is focused on the field of perovskite solar cells.

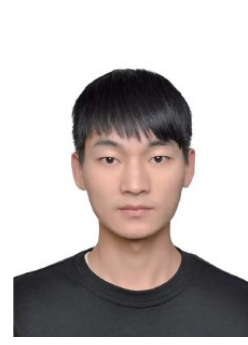

Tongle Bu received his $\mathrm{PhD}$ from WUT in 2019. He joined Okinawa Institute of Science and Technology Graduate University (OIST) as a postdoctoral fellow in 2020. He is currently working on the scalable printing of efficient and stable perovskite solar cells and modules.

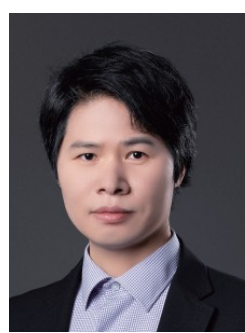

Fuzhi Huang received his $\mathrm{PhD}$ in chemistry (2009) from The University of Melbourne, Australia. Currently, he is a full professor at the State Key Lab of Advanced Technology for Materials Synthesis and Processing, WUT. His research interest is developing new materials and techniques for high-efficiency organic-inorganic hybrid perovskite solar cells. 
用于制备高效稳定钙钛矿太阳能电池的新型 spiro-OMeTAD添加剂

林志鹏 ${ }^{1,2,3}$, 李静 ${ }^{2}$, 李恒毅 ${ }^{2}$, 貌艳平 $^{2}$, 潘俊烨 ${ }^{2}$, 王超 $^{2}$, 张晓俐 $^{4}$,

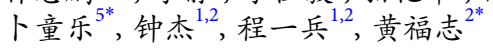

摘要 作为最常用的空穴传输材料, spiro-OMeTAD在正式结构的 高效钙铁矿太阳能电池(PSCs) 中起着重要作用. 一般来说, spiroOMeTAD是基于卤素溶剂氯苯制备的, 并且需要离子型添加剂( LiTFSI) 来提高其导电性和空穴迁移率. 然而, 这种卤素溶剂并不环 保, 而且普遍使用的Li-TFSI会影响PSCs的稳定性. 在此, 我们针对 spiro-OMeTAD开发出一种新型的p型添加剂(双氟磺酰亚胺钾, KFSI) 并且. 以无卤素溶剂四氢呋喃作溶剂将其应用于PSCs器件. 通过 该方法制备的spiro-OMeTAD薄膜具有更高的空穴迁移率、载流 子浓度以及导电性. 同时, 由空穴传输层引入的钾离子可以扩散到 钙铁矿表面使界面缺陷得到针化. 因此, 基于K-spiro薄膜的钻钛矿 太阳能电池获得了 $21.02 \%$ 的最高光电转化效率并且无迟滞现象存 在, 而且电池器件的光照稳定性和环境稳定性显著提高. 这项工作 为通过替换空穴传输材料的添加剂实现无迟滞、高效稳定的PSCs 提供了新的策略. 